\title{
La presencia social a través del feedback en entornos online de aprendizaje de idiomas
}

\author{
LOLA TORRES RÍOS \\ Campamento Norte \\ lola@campamentonorte.com \\ VANESSA RUIZ TORRES \\ Futurskolan International School of Stockholm \\ vanessa.ruiz@futuraskolan.se \\ FRANCISCO JAVIER LÓPEZ TAPIA \\ Instituto Cervantes \\ paco.lopez@cervantes.es
}

\begin{abstract}
Resumen: En el presente artículo se ofrece un recorrido teórico por los principios, estrategias e indicaciones claves a la hora de ofrecer un feedback efectivo en entornos en línea de aprendizaje de idiomas, de forma que maximicemos la presencia cognitiva, social y reflexiva de los aprendientes y los profesores. Proponemos llevar estas ideas al terreno del aula y de la práctica del aprendizaje mediante una hoja de ruta, abierta y flexible, con una serie de recursos y estrategias concretas para ofrecer y recibir retroalimentación en los contextos en línea, entornos colaborativos y personales. Se tendrán especialmente en cuenta los procesos de retroalimentación y seguimiento relacionados con la evaluación entre pares, metacognición y autoevaluación.
\end{abstract}

Palabras clave: feedback, evaluación formativa, presencia social, entornos online, feedforward.

\section{Social presence throught feedback in online language learning environments}

Abstract: This article offers a theoretical background of key principles, strategies and recommendations when offering effective feedback in online language learning environments. The main aim of the above-mentioned background is to maximize the cognitive, social and reflective presence of both learners and teachers in learning processes. The authors propose to bring these ideas to the realm of the classroom and the learning practice through an open and flexible roadmap, focusing on a handful of resources and concrete strategies to offer and receive feedback comments and to provide qualitative guidance in online collaborative and personal contexts. Follow-up processes with peer evaluation, metacognition and self-evaluation will be also analyzed.

Key words: feedback, formative evaluation, social presence, online environments, feedforward

\section{Introducción}

Aprender es un proceso secuencial que implica avances, retrocesos, dificultades y retos. Trujillo (2021) apunta que en todo proceso de aprendizaje existe una evaluación, es 
decir, una observación detallada y explícita sobre dicho proceso, pero «dicha evaluación sin feedback no sirve para nada. Sería como si alguien viniera con una checklist» (p. 1), diciendo lo que se logra hacer y lo que no, pero sin indicaciones sobre cómo mejorarlo. Por tanto, evaluación y feedback han de ir de la mano para aprender.

En el presente artículo nos proponemos reflexionar sobre la importancia del feedback en el proceso de aprendizaje en línea, especialmente para reducir el nivel de incertidumbre de los aprendientes ante su actuación, reflexión y progreso, maximizar la presencia social, cognitiva y metacognitiva del aprendizaje y, en definitiva, para aprender. Estas funciones del feedback se hacen más evidentes incluso en entornos en línea, donde aprendientes y profesores no siempre coinciden en tiempo y espacio.

La efectividad del feedback depende de quién lo ofrece y del grado de confianza que tenga con quien lo recibe; de cuándo se da, en qué momento del aprendizaje, de la clase o de la actividad y con qué frecuencia; de cómo se ofrezca según el contexto; y de cómo lo interprete quien lo recibe (Hattie y Clarke, 2018). Estas preguntas guiarán los siguientes puntos, con el objetivo de ofrecer unos pasos y recursos prácticos para ofrecer y recibir un seguimiento efectivo en el aprendizaje de idiomas en línea.

\section{El feedback como elemento clave en el aprendizaje}

Comenzamos este apartado con una reflexión sobre el feedback como elemento clave en el aprendizaje. Partimos, para ello, de una definición y unas características para un feedback efectivo, así como de una presentación de los diferentes tipos de feedback que podemos ofrecer teniendo en cuenta el tipo de actividad, el momento y el contexto, prestando especial atención a las características específicas del contexto en línea y a la importancia del feedback para monitorizar el proceso de aprendizaje en estos entornos.

\subsection{Definición y características}

Ruiz Martín (2020) define el feedback como una práctica educativa básica y natural que se basa en proporcionar al aprendiente información sobre su desempeño e indicaciones sobre cómo mejorarlo. De esta definición es destacable que se considera el feedback una práctica natural e inherente al proceso de aprendizaje que ofrecemos constantemente en el aula: cuando corregimos tareas; cuando puntuamos pruebas evaluativas; cuando nos dirigimos a un alumno que acaba de resolver una actividad o incluso cuando cambiamos nuestra expresión ante una respuesta en clase. Esa inherencia a la que hace referencia Ruiz Martín (2020) nace de la necesidad inevitable experimentada, tanto por aprendientes como por profesionales de la enseñanza, de determinar cuál es el grado de consecución de los objetivos que se proponen. Esa valoración permitirá establecer lo que puede hacerse para acercarse a esos objetivos, o darlos por alcanzados, de forma que puedan formularse otros nuevos.

Gibbs y Simpson (2009) destacan el valor del proceso de evaluación y a la inclusión del feedback en todo este proceso de manera continuada. Además, estos autores sostienen que conjuntamente con una buena selección de contenidos bien escogidos por su valor estratégico, configuran los factores clave para el bienestar del alumno. 
Tal y como comentan Guasch y Espasa (2014), el feedback no es solo una información unidireccional que proporciona el profesor al estudiante, sino un proceso de diálogo que consta de tres fases:

1. elaborar y ofrecer el feedback;

2. recibir y comprender el feedback;

3. introducir cambios para mejorar, tanto el estudiante en su proceso de aprendizaje como el profesor en relación a los contenidos, objetivos, metodología y evaluación.

Teniendo en cuenta este proceso, es importante tener en cuenta las siguientes características del feedback:

- Informativo: debe informar a todos los estudiantes de su progreso en cuanto a los objetivos y competencias, con datos sobre qué se está haciendo bien, qué se puede mejorar y cómo. A través de la información que le proporcionamos con el feedback contribuimos a que el alumno sepa cómo está desarrollando una determinada tarea y a que pueda identificar en qué momento de su proceso de aprendizaje se encuentra y cómo puede mejorarlo y continuar aprendiendo. De esta manera, podrá trabajar para cubrir la posible discrepancia existente entre su nivel de actuación real y sus objetivos de aprendizaje (Hattie y Timperley, 2007; Lozano y Támez, 2014), lo que puede llevarle a aumentar su motivación para cubrir dicha distancia y a poner en marcha estrategias más efectivas tras un proceso de reflexión (Shute, 2008).

- Centrado en el proceso de aprendizaje y orientado a la mejora (hablaremos más adelante de cómo integrar el feedforward en el aula).

- Objetivo: evitando juicios de valor.

- Motivador: centrado tanto en los aciertos como en los errores, pero guiando a todos los estudiantes a mejorar y a alcanzar los objetivos. Para que el feedback sea efectivo ha de ser gratificante, es decir, ha de provocar aprendizaje. Comentarios como "muy bien" o "necesita mejorar" ofrecerán poco margen de aprendizaje, de mejora. El feedback ha de basarse en identificar, mostrar y comentar aspectos que han mejorado en el desempeño de una acción o competencia o que pueden mejorar. Es decir, son comentarios que han de mostrar un cambio realizado o posible cambio necesario para el aprendizaje, acompañado de propuestas o pistas que ayuden al aprendiente a avanzar. En este sentido, el tipo de feedback puede provocar diferentes acciones y decisiones por parte de quien lo recibe: no hacer nada al respecto, no provocar ningún cambio o, por el contrario, hacer que el aprendiente se plantee qué puede hacer para mejorar, para aprender.

- Integrado y evaluable: asegurando que el estudiante lo incorpora a su proceso de aprendizaje. Para ello será necesario incluir estrategias que permitan saber al profesor si el estudiante ha recibido, entendido y procesado el feedback, así como las mejoras que ha decidido introducir.

La función, por tanto, del feedback, consiste en provocar cuestionamiento y revisión de lo que sabemos y aprendemos tanto fuera como dentro del aula. El feedback aportado ha de revisar las maneras de hacer, de pensar, de avanzar. Nuestras clases, por tanto, han de ocuparse principalmente de ayudar a regular el aprendizaje que ocurre en estos diferentes espacios y tiempos, y ofrecer al alumno recursos, estrategias, rutinas y hábitos que le permitan progresivamente regularse de manera autónoma. 


\subsection{Tipos de feedback}

Existen diferentes tipos de feedback en función del aspecto en el que este se centre. Vamos a centrarnos en la clasificación que realiza Ruiz Martín (2020) sobre el feedback en función de las siguientes variables:

a. Grado.

El feedback puede ser positivo o negativo. El positivo es aquel que consiste en destacar los logros alcanzados y los progresos realizados. Este tipo de feedback tiene en cuenta el esfuerzo realizado por el aprendiente y pone en valor sus resultados, que pueden cumplir o incluso superar los objetivos. Por su parte, el feedback negativo consistiría en destacar los posibles aspectos en los que el alumno puede necesitar más apoyo y acompañamiento, centrándose en identificar e informar sobre posibles áreas de mejora $\mathrm{y}$, especialmente, cómo actuar para aprender en el proceso.

b. Foco.

En lo que respecta al aspecto en el que se focaliza la acción del feedback, podemos establecer la siguiente clasificación:

1. Feedback sobre el resultado concreto de la tarea: Proporciona información concreta sobre los resultados obtenidos en una tarea. Se articula a partir de calificaciones numéricas, rúbricas o consideraciones del tipo correcto/incorrecto o apto/no apto. Proporciona información sobre el punto concreto en el que se encuentra el aprendiente con respecto a la tarea asignada y puede enriquecerse con comentarios sobre estrategias para mejorar los resultados obtenidos.

2. Feedback sobre el proceso realizado para alcanzar el resultado: No se centra tanto en la detección y corrección de aspectos susceptibles de mejora, sino que ofrece recomendaciones más holísticas y aplicables a situaciones futuras análogas.

3. Feedback sobre los procesos metacognitivos implicados en la tarea: Se centra en los procesos reflexivos sobre el aprendizaje (ej. planificación, monitorización o autoevaluación del aprendizaje), que pueden contribuir a optimizar el desempeño general de los aprendientes.

4. Feedback sobre las cualidades del alumno frente a la tarea: Se centra en la valoración de las habilidades y competencias del aprendiente en relación con la tarea. Es especialmente sensible por las implicaciones que puede tener para quien lo recibe.

c. Momento y frecuencia.

El feedback puede ser inmediato o retardado. La pertinencia de optar por uno u otro vendrá determinada por el tipo de tarea en sí y el feedback que se quiera proporcionar. Así, si lo que se pretende es valorar el resultado de una tarea, lo recomendable sería proporcionarle con la mayor inmediatez posible.

Según los trabajos de Skinner (1958) y Herrnstein et al. (1993) este feedback inmediato favorece el mantenimiento de la atención y la motivación, y que reduce la posibilidad de interiorización y posterior fosilización de los errores cometidos.

En lo que a la frecuencia se refiere, se recomienda un cierto equilibrio de forma que no sea que el feedback ni demasiado frecuente ni demasiado escaso. Un feedback muy frecuente derivaría en aprendientes muy dependientes de él para poder seguir 
avanzando; uno escaso limitaría mucho los efectos positivos del feedback en el proceso de aprendizaje y podría derivar en una cierta desorientación por parte del aprendiente.

\section{d. Modo en el que se proporciona.}

El feedback más general, y más centrado en los procesos y en la metacognición es el que ofrece mayores posibilidades de orientación al aprendiente sobre cómo optimizar su desempeño. El feedback más concreto, el que se realiza sobre el resultado de la tarea, no resulta tan relevante puesto que, además de ser menos generalizable a otras tareas, suele limitarse a apreciaciones cuantitativas. Por otro lado, si el feedback se proporciona de un modo implícito, mediante el uso de pistas que permitan al aprendiente ir descubriendo por sí mismo las respuestas correctas, estaremos favoreciendo el desarrollo de conexiones entre conocimientos y la consolidación y la estructuración de lo aprendido. Ahora bien, recurriremos al feedback explícito cuando el aprendiente no logre evocar lo aprendido.

e. Interpretación.

El feedback que proporcionamos a nuestros aprendientes debe ser comprensible e interpretable para ellos y ha de moverlos en una dirección de mejora. Tal y como afirma Ruiz Martín (2020), en ocasiones podemos encontrarnos con aprendientes sin sistemas de creencias fundados en mentalidades de crecimiento y en un sentido de eficacia positivo, es decir, de aprender lo que realmente se quiere aprender. Así pues, tengamos en cuenta las dificultades específicas que puedan presentar estos perfiles y conectemos con sus emociones y experiencias previas, de forma que el feedback pueda actuar como verdadero motor de motivación.

\subsection{Características del contexto online y especial importancia del feedback}

De Sousa Santos (2021) dice que la crisis sanitaria COVID-19 ha sido un pedagogo implacable que ha puesto de manifiesto las debilidades de nuestro estado de bienestar abriendo muchas grietas. Estas son, en general, de tipo social o económico, por ello en el contexto educativo y más concretamente en la enseñanza de lenguas extranjeras, también las podemos encontrar. Algunas de ellas están relacionadas con las condiciones laborales, otras con la tipología de cursos y clases y otras con los enfoques y metodologías didácticos. La mayoría de estas grietas ya estaban ahí antes, incluso en los contextos presenciales, pero el contexto de educación de emergencia y el auge de la enseñanza en línea la han hecho más evidente. Como dicen Carretero Gómez et al. (2021) esta situación nos ha dado la oportunidad, no solo de analizar las fortalezas y debilidades de la educación a distancia, sino también de reflexionar cómo se imparte la educación en general.

El contexto de enseñanza de emergencia a raíz de la COVID-19 puso de manifiesto desigualdades educativas relacionadas con los recursos humanos y tecnológicos y con la competencia digital de los estudiantes, profesores e instituciones. Esta situación también afectó al seguimiento, al feedback y a la evaluación en muchos cursos, cancelando o posponiendo incluso pruebas y exámenes (UNESCO et al., 2020). Sin embargo, König et al. Alabama (2020) recomiendan que, para prevenir mayores desigualdades, es necesario evaluar el progreso de los estudiantes, incluso durante una situación educativa de emergencia. Un sistema de evaluación y feedback coherente podrá facilitar un seguimiento del alumno y del curso en general, identificando progresos y dificultades, 
no solo de aprendizaje, sino relacionadas con el bienestar del alumno y del profesor, ayudando a minimizar las desigualdades educativas a las que nos hemos referido.

El aula presencial posee un carácter único y diferenciado cuyo valor radica en la comunicación cara a cara, compartiendo un mismo tiempo y espacio. En los entornos online, donde profesores y aprendientes pueden no llegar a coincidir en un espacio temporal, y donde en general existe un tiempo considerable de enseñanza y aprendizaje asíncrono, resulta más evidente y necesario maximizar esta presencia social de dichos agentes. Sin embargo, es uno de los temas que más dudas suscitan: ¿Cómo conseguir que nuestros estudiantes se sientan acompañados en su aprendizaje en un contexto en línea? ¿Cuándo y cómo podemos ofrecer feedback en favor del aprendizaje? ¿El feedback que ofrecemos es realmente aplicable? ¿Qué recursos tenemos a nuestra disposición en los entornos online? ¿Cómo los podemos utilizar para fomentar la presencia y relaciones sociales entre los agentes del aprendizaje?

En todo proceso de aprendizaje es importante hacer visibles las relaciones entre los agentes implicados. El feedback juega un papel esencial en este sentido, haciendo visible el seguimiento del progreso del alumno por parte del profesor, otro compañero o por él mismo, y ayudando a reducir el nivel de incertidumbre que puede sentir respecto a su actuación, en la realización de una actividad concreta, en su reflexión o en general en su progreso en un curso o en su proceso de aprendizaje de la lengua.

Gallardo-Cordova (2020) sostienen que la evaluación en línea no consiste en replicar a través de una cámara los procedimientos y recursos en los cursos presenciales, pero quizás también nos invitan a replantear algunos procedimientos y recursos en el aula presencial.

Los resultados del estudio de Carretero Gómez et al. (2021) muestran algunos de los principales retos en la evaluación en línea. Destacamos los siguientes:

-El seguimiento del progreso del alumno no es fácilmente observable en los contextos en línea. Destacan aspectos como las dudas y dificultades de los alumnos, la identificación de posibles problemas de seguimiento o comprensión, el seguimiento de la atención de los alumnos.

-El feedback retrasado puede afectar las experiencias de aprendizaje de los estudiantes.

- La evaluación entre pares y la autoevaluación suele estar infrautilizada.

Teniendo en cuenta esta situación y retos, ofrecemos a continuación una hoja de ruta, abierta y flexible, con diez pasos para dar y recibir un feedback efectivo en el aula de idiomas en línea, pero perfectamente adaptable a los contextos presenciales o híbridos.

\section{Hacia un feedback efectivo en entornos online de aprendizaje de idiomas}

Como hemos mencionado al inicio de este artículo, no aprendemos solos, sino en sociedad, con los compañeros, profesores, amigos y familiares. Y en este proceso, el feedback es nuestra brújula, la del docente, pero también la de nuestros estudiantes, instrumento esencial para alcanzar los objetivos de aprendizaje y llegar a los destinos esperados: el feedback forma parte del proceso continuo de aprendizaje y es el eje vertebrador de la evaluación formativa (Cano García, 2016). El feedback está 
interconectado con la experiencia de aprendizaje, no es una parcela que forme parte de la evaluación, sino del aprendizaje.

Integrar el feedback en el proceso de aprendizaje va más allá de corregir o dar la solución de una actividad: el feedback debe proponer preguntas y sugerencias que ayuden a los estudiantes a autoevaluarse. Por ello, debe contemplar propuestas y orientaciones sobre cómo pueden mejorar un determinado aspecto de su aprendizaje. Asimismo, resultará esencial plantearnos la necesidad de contar con nuevos instrumentos, estrategias y enfoques de evaluación que puedan brindarnos una imagen más completa de los aprendizajes que están ocurriendo en la actualidad en diferentes formas y contextos (Cobo, 2018) poniendo el énfasis en entornos online de aprendizaje.

En el presente apartado trataremos de desgranar las características de un feedback efectivo en entornos en línea de aprendizaje de idiomas, entendiéndolo como actividad de aprendizaje y parte de un continuum, completamente integrado tanto en la planificación, como en la gestión y en la evaluación de todo el proceso. Primero, presentamos algunas claves para entender el feedback desde la perspectiva del alumno. En segundo lugar, proponemos una hoja de ruta para el feedback en entornos online de aprendizaje. Por último, reunimos herramientas para mapear, apoyar e implementar el feedback en el aula.

\subsection{Variabilidad e impacto del feedback}

El feedback por lo común más presente en el aula de idiomas es el feedback correctivo (Kerr, 2020), cuyo objetivo es facilitar mejoras en la eficacia comunicativa de los aprendientes o resolver dudas relacionadas con el contenido lingüístico de los materiales de enseñanza y aprendizaje. Feedback is not giving, is to receiving (Hattie, 2018). Sin embargo, como hemos avanzado previamente, el aprendizaje de una lengua entraña procesos mucho más complejos en los que están implicados otros factores de índole no solo cognitiva, sino afectiva y social.

De entre todas las influencias que recibe un estudiante, una de las más poderosas es el feedback, pero también una de las más variables (Hattie y Clark, 2018). Ante esta variabilidad, existen dos factores que van a determinar su impacto y efectividad. En primer lugar, el feedback es la respuesta a tres preguntas que tienen que ver con los objetivos de aprendizaje (¿adónde voy?), el progreso (¿cómo voy a llegar?) y las consecuencias (¿cuál es el siguiente paso?). Sin este último paso no existe feedback efectivo. En este punto es importante orientar a nuestros alumnos comunicando qué acciones concretas deben tomar para que este feedback tenga un efecto real en su aprendizaje. Esta fase exige un papel activo por parte de los estudiantes. Para ello, hemos de situarlos como protagonistas del proceso de feedback: el estudiante tiene que poder hacer algo cuando recibe el feedback. Si comunicamos al estudiante qué aspectos debe mejorar, hay que prever momentos y crear oportunidades en el futuro para que puedan hacerlo.

En segundo lugar, el impacto del feedback proporcionado depende de cómo lo interpretan y reciben los estudiantes, y es aquí donde hemos de poner el foco: el feedback necesita ser recibido y bien interpretado de manera que el estudiante pueda emprender acciones de mejora. En caso contrario, no se trataría de feedback, sino de 
información, y como tal, esta sería susceptible de no mover a la realización de ninguna acción y, consecuentemente, de perderse.

Por último, un feedback efectivo es el que activa al estudiante, mapea la intención de aprendizaje, informa a los estudiantes sobre su progreso e implementa acciones de mejora previamente identificadas (Hattie \& Clark, 2018). Todo ello requiere de una determinada cultura de aprendizaje y de feedback que hemos de transmitir y enseñar a los aprendientes para que consigan un grado de autonomía cada vez mayor.

Un feedback efectivo y sostenible pasa por contar con todos los recursos humanos de los que disponemos en el aula (Hattie, 2018), no solo la autoevaluación y la evaluación del profesor, sino también la evaluación por pares. Este tipo de evaluación ayuda a que los alumnos sean conscientes de sus áreas de mejora mediante la observación de los logros y también de los errores y aspectos mejorables de sus compañeros. Existen numerosos espacios online que permiten la lectura y la retroalimentación colaborativa en grupo: tableros colaborativos como Padlet o Flipboard permiten publicar texto (oral, escrito), pero también la posibilidad de comentarlos por compañeros. Asimismo, los documentos colaborativos, como por ejemplo Google Drive, facilitan las tareas de editar, corregir y comentar un texto de manera colaborativa.

El feedback grupal ayuda a desarrollar la identidad del grupo, el feedback individual contribuye a personalizar el aprendizaje. El feedback docente es uno de los ejes vertebradores de la evaluación formativa y es esencial para el aprendizaje. Sin embargo, hemos de crear también oportunidades para que nuestros alumnos reflexionen y cooperen con sus pares a fin de favorecer una evaluación progresiva, que enseñe al estudiante a formular preguntas significativas para su aprendizaje (Brooks, Burton, y Hattie, 2021). Asimismo, el feedback ha de promover una mejora y complementarse con el feedforward, proceso que sugiere utilizar la retroalimentación para la mejora del desempeño o del aprendizaje en ocasiones futuras (Cano García, 2016). Para ello, pueden organizarse sesiones de tutoría colectiva o grupos focales utilizando muestras de los propios alumnos, o apostar por actividades de evaluación entre pares a lo largo del proceso, con recursos tales como listas de chequeo, dianas de evaluación o rúbricas.

\subsection{Herramientas para mapear, apoyar e implementar el feedback}

La evaluación formativa es el vehículo que crea oportunidades para que el feedback tenga lugar. A lo largo del proceso de aprendizaje, las herramientas de evaluación nos ayudarán a visibilizar, mapear e implementar el feedback en el aula, muy especialmente en los entornos en línea.

A continuación, proponemos recursos, herramientas y formas alternativas de evaluación destinadas a promover y guiar el aprendizaje, que incluyen la reflexión metacognitiva en el proceso de aprendizaje y, por tanto, también en la evaluación del aprendiente de lenguas. Son propuestas dinámicas y flexibles, adaptables a cualquier perfil de estudiante, curso o momento concreto del aprendizaje del alumno.

La elección de una u otra dependerá de los objetivos y circunstancias concretas de cada situación, pero pueden ofrecernos un abanico de opciones a la hora de proporcionar al alumno diversas pruebas periódicas que le ofrecen la oportunidad de responsabilizarse progresivamente de su propia evaluación y de la evaluación de sus pares. 


\section{1) Tickets de salida}

Los tickets de salida son una herramienta de evaluación formativa que nos permite comprobar, mediante una pequeña actividad o pregunta, si nuestros alumnos han comprendido o asimilado lo aprendido en una clase o lección concreta, incluyendo una reflexión por parte del estudiante (Fisher \& Frey, 2004). Usar los tickets de salida en el aula online ayuda a destacar y sintetizar los aspectos clave de cada unidad o de cada clase, y permite comprobar, tanto a estudiantes como a docentes, si se han entendido los contenidos trabajados ese día y reforzar la asimilación de este aprendizaje. En cuanto a la enseñanza, son una excelente guía docente para realizar ajustes en nuestra planificación de clase. De igual modo, los tickets de entrada son un recurso muy útil en el aula online tanto para presentar el tema y los objetivos de la sesión, como para recordar lo aprendido en clases anteriores.

En el anterior apartado, nos hemos referido a la importancia de enseñar al estudiante a formular preguntas significativas para su aprendizaje como aspecto fundamental para un feedback sostenible y efectivo. Implicar al alumno en la creación de los propios tickets de salida le ayudará a compartir la responsabilidad y a tomar conciencia de la evaluación. Se trata de una herramienta sencilla, pero que alberga un gran potencial de aprendizaje si logramos que las preguntas sean significativas. Fisher \& Frey (2004) proponen tres tipos de preguntas:

- Documentar el aprendizaje: Escribe algo que has aprendido hoy; Explica cómo utilizar lo aprendido hoy fuera del aula.

- Enfatizar el proceso de aprendizaje: No he entendido...; Escribe una duda sobre la clase de hoy; Me gustaría aprender más sobre...; Lo que me ha sorprendido más hoy es...

- Evaluar la efectividad de la instrucción: ¿Te ha gustado trabajar en pequeños grupos hoy? ¿Te han resultado claros los ejemplos?

Con recursos online se puede utilizar una pregunta de quiz individual, o una pregunta en abierto al grupo para recoger una visión general de la opinión o valoración de la clase. Recomendamos, por ejemplo, Mentimeter, que nos puede ofrecer las respuestas en tiempo real de una manera visual, en nubes de palabras o gráficos. Otra opción es ofrecer un tícket de salida en formato editable, que puedan responder e incluir en su espacio de aprendizaje, privado como blogfolios, o público como redes sociales. 


\section{Tíckets de salida}

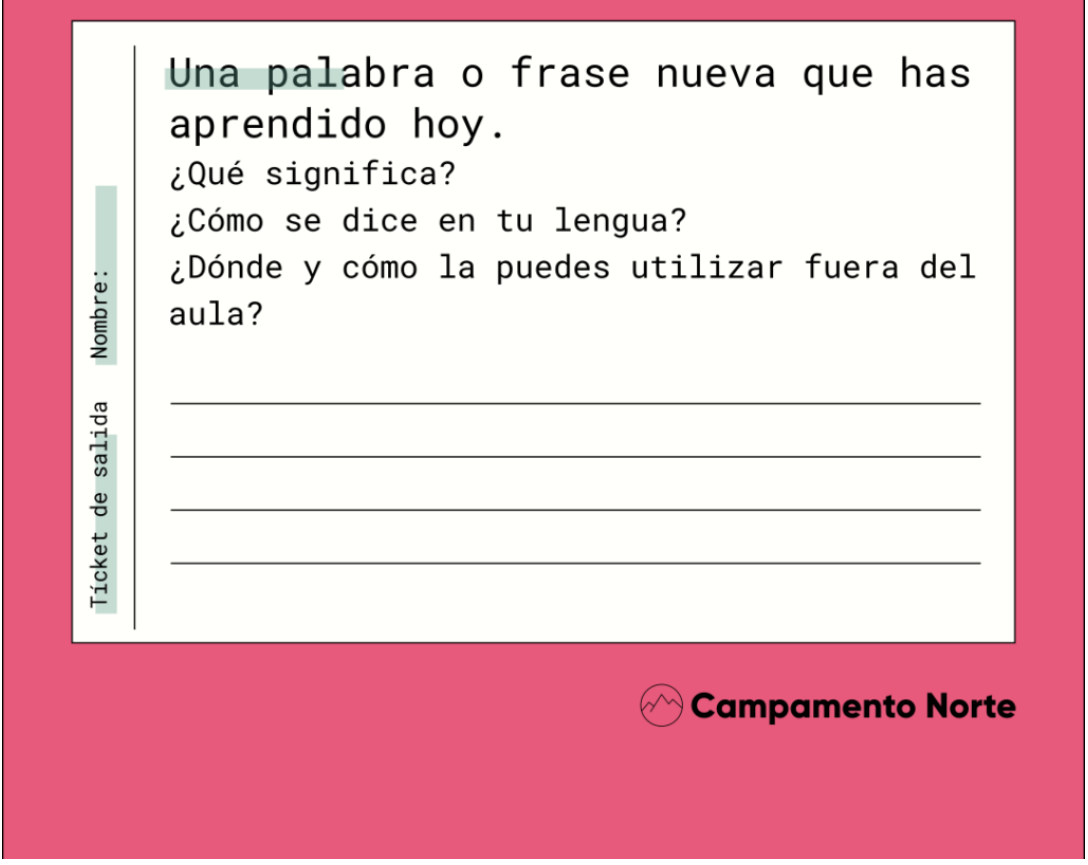

Imagen 1. Ticket de salida. Curso Evaluar para aprender, de Campamento Norte.

\section{2) Dianas de evaluación}

Las dianas son un recurso de autoevaluación basado en una representación gráfica de dicha evaluación que nos conducirá a la reflexión, a partir de una única imagen que incluye diferentes informaciones, permitiendo saber en qué aspectos del aprendizaje se debe incidir y cómo ha progresado el alumno.

El gráfico consiste en una diana con círculos concéntricos que, de dentro hacia fuera, indican el nivel de cumplimiento de cada ítem que deseemos incluir. Alrededor del círculo más amplio tendremos los nombres de los ítems y para cubrir la diana iremos indicando el número que corresponde en cada uno de ellos. Los círculos se dividen por líneas rectas creando porciones: cada porción representa un aspecto concreto a evaluar y se van coloreando cuando los resultados son positivos. De este modo, cuanta más superficie tenga color, mayor es el éxito obtenido.

Las dianas pueden emplearse de formas muy diversas y con variadas finalidades: evaluación de proyectos, de presentaciones orales, de una clase, de actividades concretas. Normalmente la diana la completa cada alumno individualmente, pero puede hacerse también en parejas o en grupos, lo cual fomenta el diálogo, la negociación, la reflexión conjunta sobre una actividad concreta y contribuye al feedback y la evaluación colaborativa. 


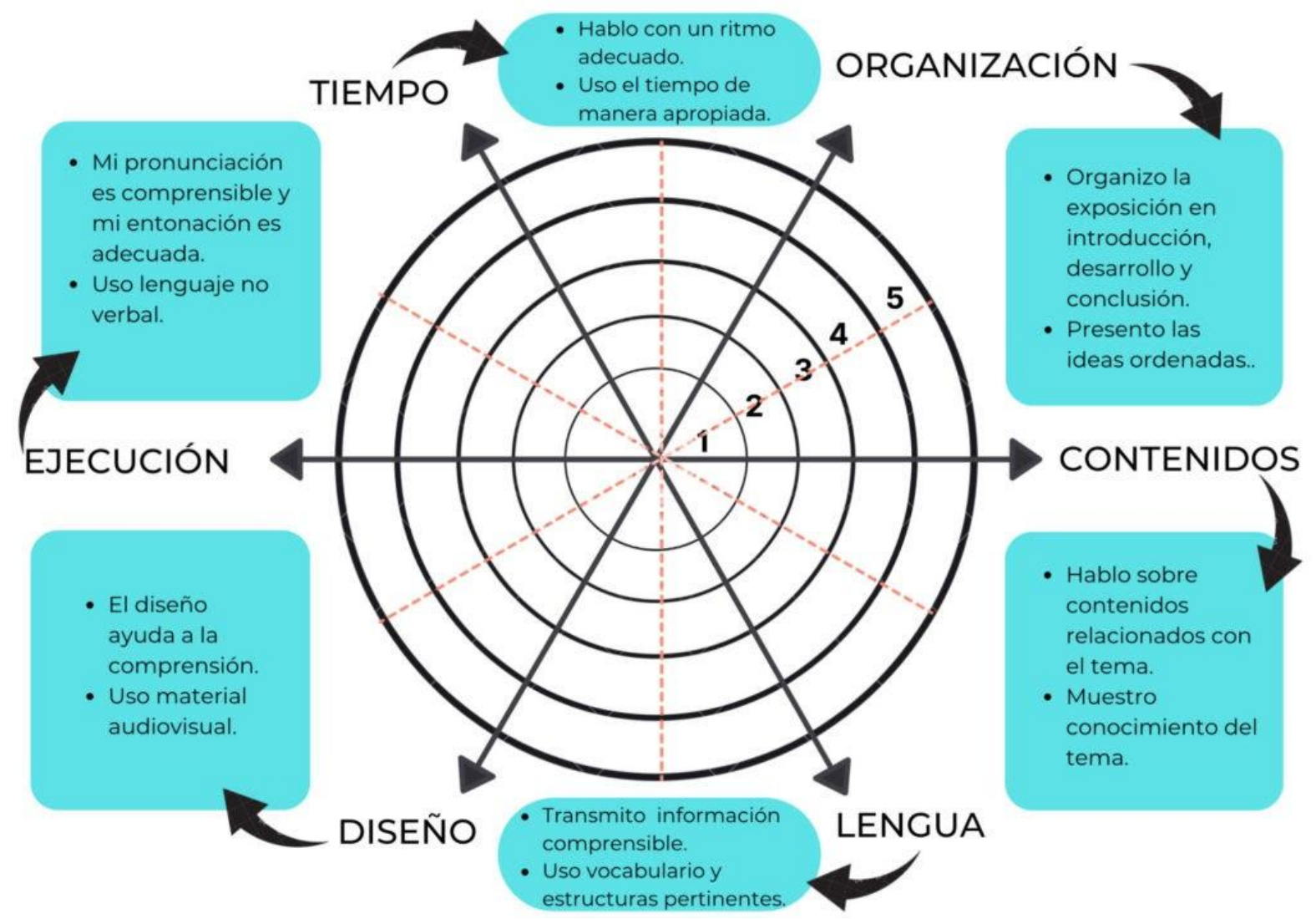

Figura 2. Diana de evaluación de la expresión oral de María Barcala en el curso Aprender en entornos híbridos (Centro Formación Profesorado Instituto Cervantes, 2021).

\section{3) Rúbricas}

La rúbrica es un documento que describe distintos niveles de aptitud, calidad o adecuación de una tarea o proyecto, dando un feedback informativo y una evaluación detallada al alumnado sobre su proceso de aprendizaje realizando dicha tarea. Es un instrumento idóneo especialmente para evaluar competencias, puesto que permite diseccionar las tareas complejas que conforman una competencia en tareas más simples distribuidas de forma gradual.

Las rúbricas pueden ser utilizadas para analizar el aprendizaje desde tres perspectivas: la evaluación docente, la autoevaluación y la evaluación entre pares (Cobo, 2018) por lo que, bien diseñadas y monitorizadas en el aula, son una poderosa herramienta de evaluación colaborativa. Se trata de una herramienta que aumenta la transparencia de la evaluación y reduce la subjetividad de la evaluación entre los diferentes agentes evaluadores $y$, en consecuencia, la percepción de justicia en dichos procesos. Pueden emplearse para dar feedback, casi inmediato, a los estudiantes, en el marco de una evaluación formativa y continuada.

Al tratarse de un documento para trabajar con los alumnos, es preferible usar un lenguaje claro y sencillo, libre de tecnicismos que puedan entorpecer su comprensión. Asimismo, es importante la precisión y la claridad al describir cada aspecto y su nivel de ejecución para que nuestros estudiantes sean conscientes de lo que estamos evaluando y entiendan por qué los situamos en un nivel y no en otro. En entornos de 
aprendizaje en línea, recomendamos hacer de la rúbrica una herramienta visual, manipulable y personalizada que permita al estudiante monitorear la actividad y su aprendizaje.

\section{4) Cuestionarios}

Los cuestionarios son herramientas de evaluación que nos pueden servir para diversos propósitos y en diferentes momentos del proceso de aprendizaje y enseñanza. Al final de una unidad o actividad para comprobar lo que se ha aprendido y especialmente para ofrecer una nueva oportunidad al aprendiente de encontrarse con el aprendizaje. Al inicio de un curso, unidad o actividad, como diagnóstico de lo que sabe el alumno o la clase en general sobre un tema. En cualquier momento del proceso, para conocer la opinión y feedback de los alumnos o compañeros sobre un tema o sobre una actividad, unidad o curso.

Entre sus ventajas, se encuentra la de resultar una herramienta sencilla de suministrar, realizar y de obtener información, incluso con estadísticas y gráficos que permiten al alumno y al profesor tener una idea visual de los resultados, individuales o colectivos. Los cuestionarios nos permiten personalizar el itinerario del cuestionario según las respuestas, lo cual es muy útil a la hora de ofrecer feedback pertinente a cada estudiante. Existen muchas opciones digitales para realizar cuestionarios online: Survey Monkey, Doodle, Typeform o Mentimeter. Proponemos Google Forms, por la posibilidad que nos ofrece para descargar todos los resultados directamente a un Excel, visualizar los resultados y personalizar el proceso.

Los alumnos se pueden involucrar en su creación y diseño, favoreciendo el trabajo colaborativo y la evaluación compartida y entre pares. Para ello es imprescindible introducir los cuestionarios en el aula como una actividad periódica.

\section{5) Estaciones de aprendizaje}

Las estaciones de aprendizaje son una dinámica dentro del aula (presencial u online a través de diferentes salas o entornos online) que consiste en crear espacios dentro del aula, en forma de circuito de aprendizaje, en el que los alumnos van pasando por diferentes estaciones en grupos, en cada una de las cuales encontrarán una hoja de ruta con las pautas para la realización de una actividad.

Los beneficios de esta propuesta son numerosos. Favorece el colaborativo y la atención a la diversidad, al trabajar en pequeños grupos en los que se implica a todos los estudiantes. Posibilita una atención y seguimiento más individualizado de la actuación y progreso de cada estudiante. Favorece la revisión del aprendizaje desde una perspectiva general e incluye rutinas y dinámicas de pensamiento para valorar, dar feedback, comparar y tomar decisiones.

\section{6) Reflexión encapsulada en redes sociales}

Los diarios de aprendizaje y los portafolios lingüísticos del aprendiente de lenguas son dos recursos óptimos para trabajar la reflexión sobre el aprendizaje.

Con respecto a los diarios de aprendizaje, podemos pedir a los alumnos que escriban un diario utilizando diferentes aplicaciones y redes sociales que pueden enriquecer sus funciones mediante la incorporación de elementos audiovisuales que correspondan a 
cuatro tipos de muestras: de aprendizaje, de lengua, de estrategias utilizadas y sobre emociones relacionadas con el aprendizaje de la lengua meta.

En el caso de los portafolios de lenguas, podemos establecer tres momentos de entrega a lo largo del proceso de aprendizaje con su correspondiente feedback por parte del docente, del propio estudiante, de un compañero o combinando estos feedback entre sí. Para ello, resultará de gran ayuda una rúbrica compartida y consensuada en el aula.

\section{Feedback, motivación y emociones}

Tal y como apuntan, Batalla-Busquets et al. (2014) en su estudio, «podemos afirmar que el feedback personalizado acerca la relación entre los docentes y los estudiantes, y que tiene efectos que favorecen el aprendizaje y motivan al estudiante» (p. 98). El feedback, pues, se erigiría como una de las prácticas más poderosas de las que disponemos en los procesos de enseñanza y aprendizaje. Entendemos que esta afirmación cobra más sentido aún en entornos online en los que la presencia de los diferentes actores de los mencionados procesos puede no resultar tan evidente.

Independientemente del entorno en el que se proporcione el feedback, este ha de ser ser comprensible y fácilmente interpretable por ellos. Un feedback efectivo ha de mover y acompañar hacia el desarrollo constante y hacia la mejora. Es decir, ha de convertirse en fuente de motivación y, en definitiva, en motor de aprendizaje.

Así pues, en este último punto de nuestro artículo, compartimos algunas reflexiones sobre el feedback y sobre su impacto en la motivación. Así mismo, indagaremos en la dimensión afectiva, refiriéndonos específicamente a las diferentes emociones que pueden experimentar nuestros aprendientes durante las tres fases de las que, como indicábamos en el primer punto, se compone el feedback: elaboración y proporción del feedback, recepción e interpretación de este, $\mathrm{y}$, finalmente, su implementación.

En una primera fase de elaboración y proporción del feedback, será importante medir bien las palabras con las que lo articulamos. No podemos olvidarnos de que el feedback se compone, en gran medida, de valoraciones, y de que estas no son siempre fáciles de encajar ni en el educativo ni en muchos otros ámbitos vitales. Por ello, habría que formularlo siempre desde la crítica constructiva y en términos de oportunidad. Solo en este caso, como indica Ruiz Martín (2020), el feedback tendrá la capacidad de estimular emocionalmente a su receptor, asegurando que este le prestará la mayor atención posible y que, en consecuencia, podrá recordarlo mejor cuando tenga que recurrir a él en el futuro. En este sentido, cobra especial interés la noción de feedback formador, que fomenta la autonomía del aprendiente y favorece el ajuste y regulación de la enseñanza y la del propio aprendizaje (Pérez Morales, 2008).

Por otro lado, ejercer la empatía, conectando con nuestras propias experiencias como aprendientes y receptores de feedback podría sernos de gran ayuda. Cobra especial protagonismo nuestra capacidad de ponernos en la piel del otro. Desde esa empatía, podrán generarse espacios de diálogo que sean traducibles en acciones de apertura, de reflexión, fundamentales en el feedback y en cualquier otro proceso de los que componen el aprendizaje (Barria Díaz, 2016).

Unida a la empatía, se encuentra también la idea de la personalización del feedback a la que ya hacían referencia anteriormente Batalla-Busquets et al. (2021): un feedback que 
no fuera individualizado, es decir, que no tuviera en cuenta las circunstancias específicas de cada una de las situaciones de aprendizaje en las que se proporciona y de cada una de las personas implicadas, podría derivar en falta de conexión, desánimo o pérdida de interés. Se trata, en definitiva, de tener en cuenta todo aquello que ya conocemos sobre la persona a la que nos dirigimos, sus creencias. Serán, pues, estas últimas, las que determinarán su capacidad para beneficiarse del feedback y las que le transmitirán, en cierta manera, sensaciones de atención y de cuidados respecto a quien le transmite el feedback.

Así mismo, el impacto y la efectividad del feedback serán diferentes y únicos en función, precisamente, de esas cuestiones personales y de factores afectivos. Si no se ha creado previamente un entorno emocional seguro en el que proporcionarlo, habrá que trabajar primero en llegar a él antes de que se produzca su transmisión. Según Ruiz Martín (2020), se trata de fomentar en el estudiante expectativas positivas, que fomenten su confianza en sí mismo y su sentido de autoeficacia respecto a la implementación de las acciones que ha de llevar a cabo como parte de su proceso de mejora.

En lo que se refiere a los niveles de desempeño de nuestros aprendientes de idiomas y la preferencia por un tipo de feedback u otro, Ruiz Martín (2020) apunta que los expertos coinciden en que, por ejemplo, en los niveles iniciales es importante que predomine el feedback positivo, puesto que este potenciará la disposición del alumnado a seguir mejorando. En niveles superiores, sin embargo, los aprendientes han alcanzado ya un grado considerable de autoconocimiento y han desarrollado una mayor autonomía. Además, se tiene un conocimiento más claro y certero de los objetivos que quedan en el horizonte. Por ello, en estos últimos, el feedback negativo podría resultar motivador.

En cualquier caso, las valoraciones menos positivas deberán realizarse sobre cuestiones que pudieran ser realmente mejorables puesto que, de lo contrario, estaríamos provocando frustraciones, más que fomentando aprendizajes. En esta misma línea, estudios, como los realizados por Freedberg et al. (2016) en torno a la efectividad del feedback negativo, concluyen que este, cuando es proporcionado sobre procesos o estrategias y no sobre los resultados obtenidos, tiene un gran potencial transformador, puesto que animará a la persona que lo recibe a reorientar su esfuerzos y concienciar sobre el margen de mejora con el que se cuenta.

Habrá, pues, que guiar a nuestro alumnado para que sepa gestionar el feedback negativo proporcionado, llevando a cabo un seguimiento pormenorizado de las acciones que está realizando para mejorar. Sería necesario, igualmente, que las valoraciones negativas fueran acompañadas de soluciones, que permitieran hacer frente a situaciones desfavorables y que favorezcan actitudes de resiliencia.

Otra cuestión clave relacionada específicamente con el feedback negativo es saber transmitir a nuestro alumnado que el error es inherente al proceso de aprendizaje, que equivocarse significa inevitablemente aprender. Ahora bien, no hemos de olvidarnos, en ningún caso, de que los errores tienen, en ocasiones, un impacto emocional negativo en nuestros aprendientes. Los errores también pueden provocar vergüenza, frustración, limitación y, por lo tanto, pueden ralentizar nuestro desempeño de una actividad o, de forma más general, nuestro aprendizaje. De nuevo, el sistema de creencias de profesores y estudiantes pueden condicionar las actitudes hacia el error. 
Ruiz Martín (2020) señala que ayudaría mucho que los errores que más suelen importar a nuestros estudiantes, que son principalmente los que se dan durante los diferentes procesos evaluativos que desarrollamos dentro y fuera de las aulas presenciales o online, no se percibieran como irremediables. Esta percepción de irremediabilidad es la que se da cuando la evaluación se limita al final del proceso de aprendizaje, es decir, cuando esta busca, exclusivamente, constatar el nivel alcanzado sin ofrecer, a continuación, oportunidades para mejorar en el mismo tipo de tarea o en el mismo tema.

Cuando la evaluación se integra en el propio proceso de aprendizaje, en oportunidades de bajo riesgo que permiten cometer y reparar errores anteriores en la siguiente oportunidad, los estudiantes pueden empezar a entender que cometer un error no es señal de su falta de habilidad, sino que verdaderamente se trata de algo inherente al proceso de aprendizaje como apuntábamos con anterioridad. De lo contrario, la evaluación se percibe como una constatación de una supuesta habilidad inamovible, no como la constatación de un estado transitorio y genera desesperanza y, de nuevo, frustración. Por todo ello, es esencial proporcionar feedback durante el proceso (diferentes entregas de una tarea, sesiones de feedforward, tareas reflexivas), fomentando la capacidad de autorregulación del estudiante.

Independientemente del grado de éxito obtenido por parte de los aprendientes en torno a los objetivos predeterminados, es igualmente aconsejable proporcionar un feedback que focalice en los esfuerzos realizados, entendiendo estos esfuerzos como signos inequívocos de crecimiento, más que en las propias habilidades. Podría pensarse que estas últimas son innatas, es decir, o se tienen o no se tienen y que, a corto plazo, resultan complejas de mejorar. Lo recomendable es limitar el uso del feedback que alude a las cualidades del alumno frente a la tarea realizada y focalizar siempre en la tarea y en el camino realizado para resolverla.

Queremos referirnos también al hecho de que, a pesar de tener en consideración todos los factores que se han ido exponiendo, los efectos que el feedback puede provocar en nuestros aprendientes son impredecibles. Se requiere, por nuestra parte, una reflexión sobre si es verdaderamente necesario aportarlo, y la cuestión se vuelve aún más delicada si el feedback es transmitido en público o en privado.

En lo que se refiere a los ámbitos públicos y privados, los orígenes culturales del alumnado cobran una especial importancia. Hay culturas como la china, en las que el "perder la cara" por haber recibido un feedback negativo en público, delante de los compañeros, podría resultar verdaderamente traumático. Hay autores como William (2011) que recomiendan no proporcionar feedback en público a no ser que sea absolutamente indispensable.

Por último, nos referiremos a las notas, o calificaciones, que solemos asignar cuando realizamos tareas o pruebas evaluativas. Más allá de que haya aprendientes para los que las calificaciones puedan ser estimulantes, proponemos que vayan siempre acompañadas de explicaciones sobre qué puede hacer el aprendiente para mejorar. Cuanto más descriptivo y detallado sea el feedback, mucho mejor.

Tal y como afirma Ruiz Martín (2020) la contribución de las notas al aprendizaje es ciertamente reducida, ya que son un tipo de feedback que no responde a una de las preguntas más importantes a las que ha de responder el feedback efectivo. 
Igualmente, y si se opta por sistemas de evaluación basados en calificaciones numéricas, habría que evitar que se otorguen únicamente cuando el proceso de aprendizaje que se está realizando se da por finalizado. Proporcionar feedback a los aprendientes de manera continuada es primordial y, por ello, resulta apropiado el uso de tareas o pruebas evaluativas periódicas, que generan nuevamente sensaciones de control, atención y cuidados. De este modo, las posibles actitudes negativas de los alumnos hacia el feedback se verían reducidas y su atención al feedback aumentaría.

En lo que respecta a las calificaciones, incluimos dos propuestas concretas aportadas por Ruiz Martín (2020) y otra tercera, que añadimos nosotros, que resumirían las ideas contenidas en estos últimos párrafos:

Propuesta 1: Otorgar calificaciones de forma rutinaria en todas las actividades y tareas de aula. De este modo, estaríamos otorgándoles a todas ellas valor académico. Sin embargo, lo más interesante en esta propuesta sería que, tras recibir la nota, los estudiantes tuvieran la oportunidad de repetir la actividad y mejorarla en caso de que fuera necesario.

Propuesta 2: Realizar pruebas evaluativas que se corrigieran dos veces. En una primera corrección, nos limitaríamos a aportar feedback (indicaciones y pistas sobre cómo mejorar) $\mathrm{y}$, en una segunda, incluiríamos las calificaciones que correspondieran en cada caso.

Propuesta 3: Mostrar retos optativos e insignias para mejorar la nota a aquellos estudiantes que vayan más avanzados (y especificar cómo), sin penalizar a ninguno.

\section{Conclusiones}

A modo de conclusión general, proponemos un feedback centrado en procesos que en resultados, que se haga evidente en diferentes momentos, de forma que, a pesar de la aparente independencia y lejanía que suele asociarse a los entornos online o híbridos, se convierta en garante constante de presencia social de todos los implicados. Aprovechemos también esa constancia, para que se articule a partir del análisis de todas las variables dadas y factores humanos y personales, característica esta, a nuestro juicio, indispensable para que el feedback se oriente hacia la mejora y el crecimiento continuos.

\section{Bibliografía}

BARRIA DÍAZ, Daniela. 2016. «Empatía como emoción emergente en el proceso de enseñanza y aprendizaje», Paideia surcolombiana, 21, 112-123. Recuperado el 19/12/21, de: https://dialnet.unirioja.es/descarga/articulo/7836173.pdf.

Batalla-Busquets, Josep Maria, Plana ERta, Dolors, Martínez ArgüElles, María José. 2014. "La importancia del feedback en un entorno virtual de aprendizaje». Oikonomics, Universitat Oberta de Catalunya, 1, 93-100. Recuperado el 19/12/21, de: https://www.researchgate.net/publication/335427483_La_importancia_del_feedback_en _un_entorno_virtual_de_aprendizaje 
Brooks, Cameron, Burton, Rochelle y HATTIE, John. 2021. «Feedback for learning». En Building Better Schools with Evidence-based Policy, Londres: Routledge, 65-70.

CANO, Elena. 2016. «Del feedback al feedforward». En N. Cabrera y R. Mayordomo (ed.), El feedback formativo en la universidad. Experiencias con el uso de la tecnología. Barcelona: LMI. (Colección Transmedia XXI), 32-40. Recuperado el 19/12/21, de: https://www.researchgate.net/publication/301636745_El_feedback_formativo_en_la_un iversidad_Experiencias_con_el_uso_de_la_tecnologia

Carretero Gómez, Stephanie, Napierala, Joanna., Bessios, Adonis, Mägi, Eve, Pugacewicz, Agniezska, RAnieri, Maria, TriQuet, Karen, LOMBAERTs, Koen, Robledo Bottcher, Nicolas, Montarani, Marco y GonZÁlez VÁzQueZ, Ignacio. 2021. «What did we learn from schooling practices during the COVID-19 lockdown», Publications Office of the European Union, EUR 30559 EN, Luxembourg.

Cово, Cristóbal. 2018. La innovación pendiente. Reflexiones (y provocaciones) sobre educación, tecnología y conocimiento. Recuperado el 19/12/21, de: https://digital.fundacionceibal.edu.uy/jspui/bitstream/123456789/159/1/La_innovacion_ pendiente.pdf

GIBBS, Graham y SiMPSON, Claire. 2009. «Condicions per a una avaluació continuada que afavoreixi l'aprenentatge». Quaderns de Docència Universitària. Barcelona: ICE de la Universitat de Barcelona / Editorial Octaedro.

GuAsCh, Teresa y EspasA, Anna. 2014. Guia pel disseny d'un feedback formatiu $i$ dialògic. Universitat Oberta de Catalunya/Grup de recerca EdOnline. Recuperado el 19/12/21, de: http://openaccess.uoc.edu/webapps/o2/bitstream/10609/37801/1/guia feedback_formati u_dialogic_2014_def_tguaschp_aespasa.pdf

HAtTIE, John. y CLARK, Shirley. 2018. Visible Learning: Feedback. Londres: Routledge.

KERR, Philip. 2020. Giving feedback to language learners. Part of the Cambridge papers in ELT series. Cambridge: Cambridge University Press.

RUIZ MARTíN, Héctor. 2020. ¿Cómo aprendemos? Una aproximación científica al aprendizaje y la enseñanza (1a edición). Barcelona: Editorial Graó.

SousA SANTOS, Boaventura. 2020. La cruel pedagogía del virus. Madrid: Ediciones Akal.

TRUjILlO, Fernando. 2021. «Enseñar en la era digital. Entrevista a Fernando Trujillo». Educaixa. Recuperado el 19/12/21, de: https://educaixa.org/es/-/entrevista-a-fernandotrujillo-

WiLLIAM, Dylan. 2011. Embedded formative assessment. Solution Tree Press. 